\title{
Interchange between collagenous and lymphocytic colitis in severe disease with autoimmune associations requiring colectomy: a case report
}

\author{
T E Bowling, A B Price, M Al-Adnani, P D Fairclough, N Menzies-Gow, D B A Silk
}

\begin{abstract}
Background-Collagenous colitis and lymphocytic colitis present with a similar clinical picture. Whether these conditions are separate entities or whether they represent different pathological stages of the same condition is an unresolved issue. Patient-This is a case of collagenous colitis following a fulminant course in which a colectomy was necessary. In the operative specimen the thickened collagen plate, which had been present only two weeks preoperatively had been lost and the pathology was of a lymphocytic colitis. Six months postoperatively this patient developed a CREST syndrome and primary biliary cirrhosis.

Conclusions-This case shows the lability of the collagen plate and the common ground between collagenous and lymphocytic colitis, and presents evidence that these two conditions are different manifestations of the same disease. It also describes for the first time an association between collagenous colitis and CREST syndrome and primary biliary cirrhosis. (Gut 1996; 38: 788-791)
\end{abstract}

Keywords: collagenous colitis, lymphocytic colitis, interchange, colectomy, autoimmunity.

Collagenous colitis and lymphocytic colitis are recently described inflammatory disorders of the colon. ${ }^{12}$ Clinically, they are characterised by a history of fluctuating watery diarrhoea but with normal appearances at barium enema and colonoscopy. The clinical course of both conditions is benign and there has not been, to our knowledge, any report of either condition following a fulminant course necessitating colectomy. ${ }^{3}$ They do differ in certain histological abnormalities, most notably the thickened subepithelial collagen plate that occurs in collagenous but not lymphocytic colitis, ${ }^{4}$ but in both a raised number of intraepithelial lymphocytes has been reported. ${ }^{4}$ This last fact and the uneven distribution of the collagen plate both within any series of biopsy specimens and between interval series has led to considerable debate whether these are two separate conditions, or whether they represent different stages of the same disease process. ${ }^{4-11} \mathrm{We}$ report a case of collagenous colitis following a fulminant course necessitating emergency colectomy, demonstrate the lability of the collagen plate, and the common ground between collagenous and lymphocytic colitis.

\section{Case report}

A 46 year old white woman was referred in December 1992 with a three month history of persistent diarrhoea after a course of amoxycillin for an upper respiratory tract infection. Ten years previously she had been investigated for diarrhoea with a barium enema, barium meal, and jejunal biopsy. No diagnosis was made, and a few months later the diarrhoea stopped spontaneously. There was no other relevant past medical history. At the initial consultation all her blood tests were normal, but three day faecal weights were abnormal at 271 g/day (normal $<200$ ). Faecal fat was normal, stool cultures for enteric pathogens and Clostridium difficile toxin were negative. A colonoscopy was macroscopically normal, but biopsy specimens showed clear evidence of a diffuse collagenous colitis (Fig 1). She was given salazopyrine (1 $\mathrm{g}$ three times a day) and prednisolone (10 mg/day), and for the next six weeks her symptoms gradually improved. In early February 1993, however, she became acutely unwell with a fever, severe diarrhoea, and cramping abdominal pains. Her blood results were normal, except for a neutrophil leucocytosis of $18.5 \times 10^{9} / 1$ (normal $<11 \times 10^{9} /$ ), and her faecal weights had increased to 374 g/day (Fig 2). Plain abdominal $x$ ray showed

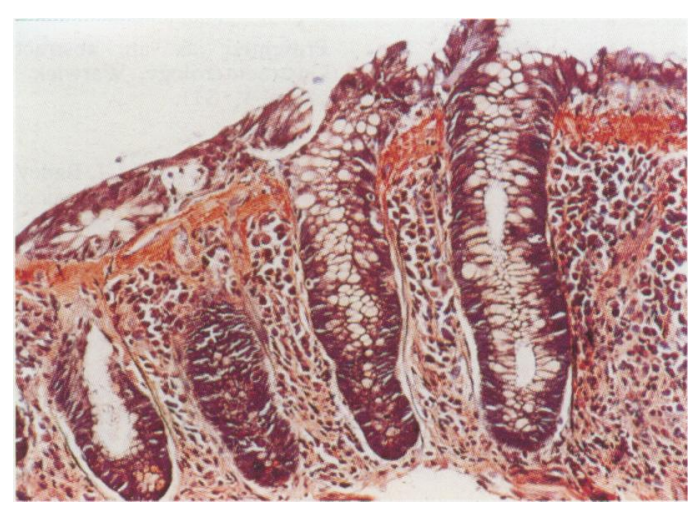

Figure 1: A phosphotungstic acid stain shows the thickened collagen plate of up to $25 \mu \mathrm{m}$, which is accompanied by a low grade colitis in the underlying lamina propria. 


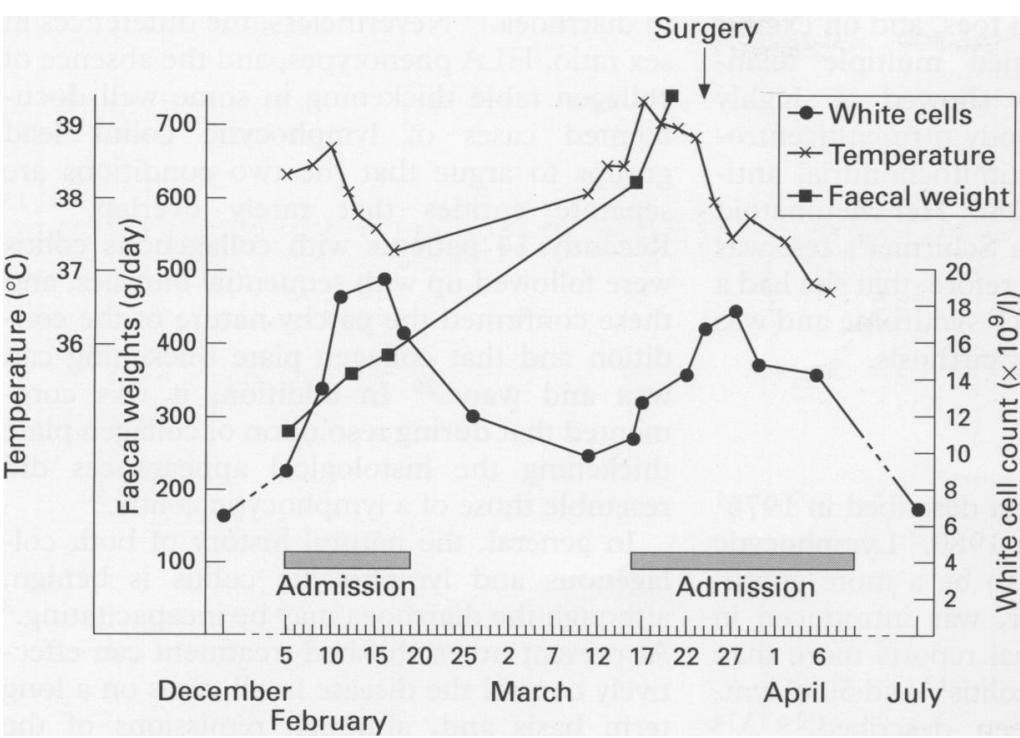

Figure 2: Clinical course charting faecal weights, temperature, and white blood cell count.

evidence of a total colitis. The macroscopic appearances at a second colonoscopy showed mild patchy inflammation with loss of the vascular pattern throughout the colon (Fig 3). Biopsy specimens again showed an active total collagenous colitis and inflammation extended into the terminal ileum. She improved slowly with high doses of corticosteroids (40 mg prednisolone/day), azathioprine (100 $\mathrm{mg} /$ day), and salazopyrine ( $1 \mathrm{~g}$ three times a day) and was discharged in late February. Four weeks later she again became acutely unwell with a fever, and plain abdominal $x$ ray again showed a total colitis and mucosal oedema with no intestinal dilatation. Her white cell count was $17 \times 10^{9}$ and faecal weights $745 \mathrm{~g} /$ day. All her other blood tests, including erythrocyte sedimentation rate and albumin remained normal. A rectal biopsy at this time showed nonspecific inflammatory changes only with no features of collagenous colitis. On this occasion she did not respond to medical treatment, and remained acutely unwell with profuse diarrhoea, fever, abdominal tenderness, and leucocytosis. She therefore underwent an emergency colectomy and ileostomy. At surgery the serosal surface of the colon was hyperaemic but, unlike that to be expected of an acute pancolitis, the macroscopic appearance of the wall and the mucosal surface of the resected colon
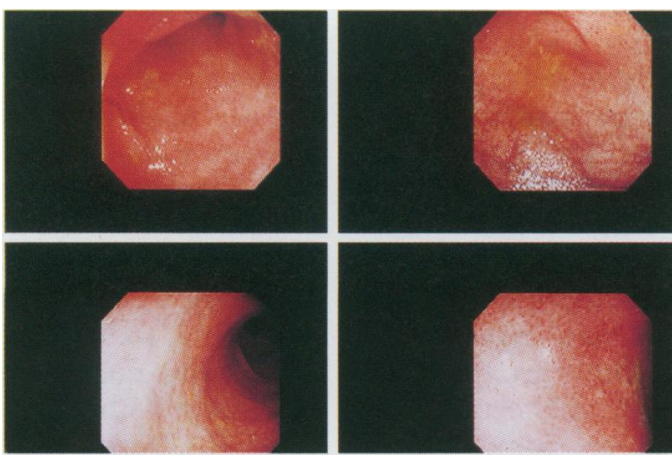

Figure 3: Colonoscopy appearances in the terminal ileum (top right), caecal pole (top left), ascending colon (bottom right), and hepatic flexure (bottom left). The colon is diffusely abnormal with loss of vascular pattern and granularity.

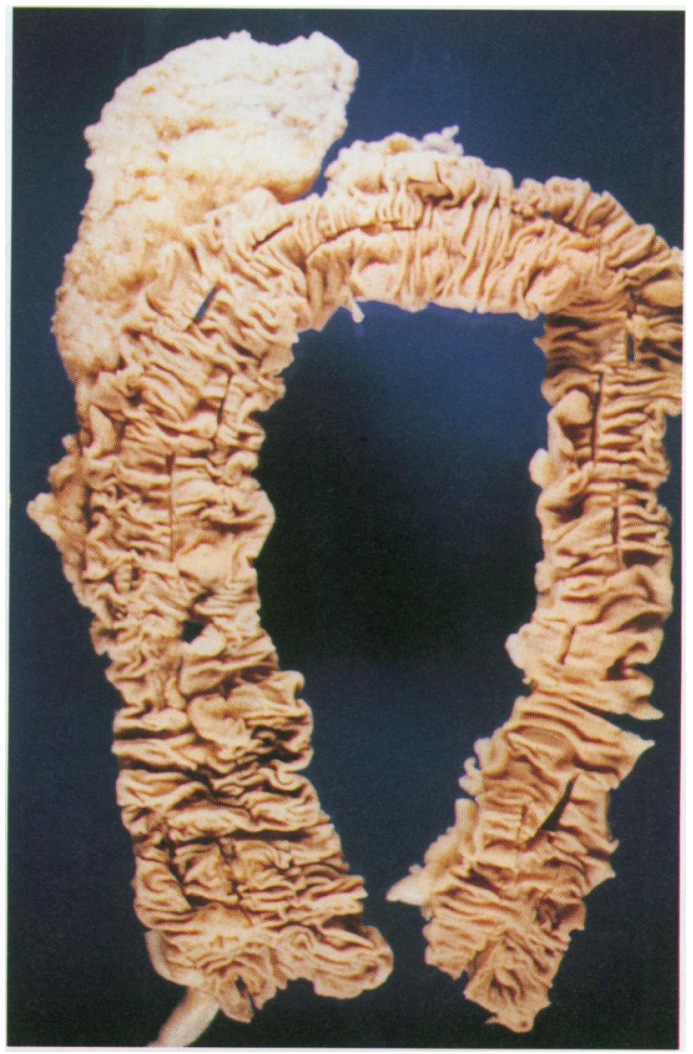

Figure 4: The colectomy specimen with no gross abnormality visible. (The mucosal defects are the sites from which microscopy blocks were selected.)

were unremarkable (Fig 4). Extensive sampling only showed focal areas with a raised intraepithelial lymphocyte count of up to 40 per 100 epithelial cells (normal <5) superimposed on a background count of 14-18 per 100 epithelial cells. Occasional branched crypts, indicative of previous damage, were present (Fig 5). Neither a thickened collagen table nor signs of a severe colitis were seen. Postoperatively she made an excellent recovery, and had her ileostomy reversed in July 1993. The intraepithelial surface lymphocyte count in the rectum at the time of reversal was still above normal at 15-20 per 100 epithelial cells. The ileal count was normal.

From a gastrointestinal point of view she remained well, but six months after the ileostomy was reversed it was noted that her $\gamma$-glutamyltransferase and alkaline phosphatase were marginally increased. In addition she was complaining of arthralgia, myalgia, dry

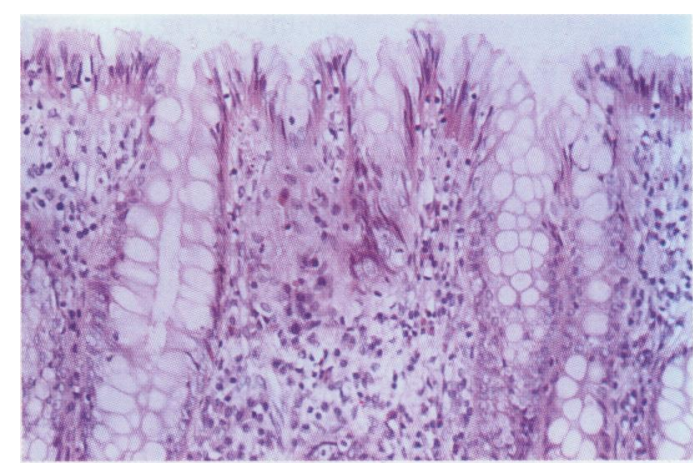

Figure 5: Microscopy from the colectomy illustrates a raised intraepithelial lymphocyte count (about 40 of 100 colonocytes) but no other abnormality, in particular no residual evidence of a thickened collagen plate. 
eyes, and cold fingers and toes, and on examination she had developed multiple telangiectasia. Investigations showed a highly positive antinuclear antibody titre, anticentromere antibodies, and antimitochondrial antibodies with a titre of $1: 1280$. Her rheumatoid factor was negative and a Schirmer's test was dry. It was concluded, therefore, that she had a mild version of the CREST syndrome and was developing primary biliary cirrhosis.

\section{Discussion}

Collagenous colitis was first described in $1976^{1}$ and microscopic colitis in $1980 .^{2}$ Lymphocytic colitis, thought by some to be a more appropriate term for the latter, was introduced in $1989 .{ }^{4}$ Since these original reports more than 230 cases of collagenous colitis ${ }^{12}$ and 50 of lymphocytic colitis have been described. ${ }^{2} 61314$ These two conditions have many features in common. Both present with cramping abdominal pain and watery diarrhoea, caused by decreased colonic fluid absorption. ${ }^{14-16}$ In both the colon appears normal at colonoscopy and on barium enema, and in both the diagnosis can only be made by biopsy. Differences between the two include a smaller female predominance in lymphocytic colitis ${ }^{4}$; an increase in HLA A1 and DRw 53, and a decrease in HLA A3 phenotypes in lymphocytic colitis ${ }^{13} 17$ (there are no changes in HLA phenotype in collagenous colitis from the normal population ${ }^{17}$ ). There are also differences in histological appearance. The presence of increased intraepithelial lymphocytes, often above 20 per 100 epithelial cells (normal <5), is mandatory to the diagnosis of lymphocytic colitis, but it can accompany collagenous colitis. ${ }^{411}$ There were an average of 14 lymphocytes per 100 epithelial cells in the two presenting colonoscopic series, the predominant cell being the neutrophil polymorph, reflecting the severity of the colitis at these times. Obviously in collagenous colitis there has to be a thickened subepithelial collagen table, with widths greater than 7-10 $\mu$ generally regarded as significant. ${ }^{11} 18$ There is also reported to be greater eosinophilic infiltration ${ }^{4}$ and increased surface epithelial damage in collagenous colitis than in lymphocytic colitis. ${ }^{4}$ However, as the abnormality of collagenous colitis can be restricted to the right colon, be intermittently present and, as stated, be accompanied by a raised intraepithelial lymphocyte count during its natural history, a watertight distinction between the two entities will require the prolonged study of appropriate patient groups or the identification of distinct aetiologies.

To date some groups have reported histological progression from lymphocytic to collagenous colitis ${ }^{5-8}$ and, along with the clinical and histological similarities between the two conditions, subscribe to the view that they do represent the same disease. Other groups argue that if this was the case and lymphocytic colitis did progress to collagenous colitis, there should be a correlation between collagenous colitis and the severity of diarrhoea - no such correlation has been found. ${ }^{18}$ Moreover, a thickened collagen plate does not always lead to diarrhoea. ${ }^{19}$ Nevertheless, the differences in sex ratio, HLA phenotypes, and the absence of collagen table thickening in some well documented cases of lymphocytic colitis lead groups to argue that the two conditions are separate entities that rarely overlap. ${ }^{9-11} 13$ Recently 14 patients with collagenous colitis were followed up with sequential biopsies, and these confirmed the patchy nature of the condition and that collagen plate thickening can wax and wane. ${ }^{20}$ In addition, it was commented that during resolution of collagen plate thickening the histological appearances did resemble those of a lymphocytic colitis. ${ }^{20}$

In general, the natural history of both collagenous and lymphocytic colitis is benign, although the diarrhoea may be incapacitating. ${ }^{3}$ At present no established treatment can effectively control the disease in all cases on a long term basis and, although remissions of the disease and disappearance of the collagen band have been described, ${ }^{520-22}$ relapse of both symptoms and histological abnormalities is common. ${ }^{23} 24$ Recently it has been shown that diverting the faecal stream results in the disappearance of the collagen plate, suggesting a luminal toxin may have an aetiological role. ${ }^{25}$

An association between antibiotic treatment and collagenous colitis has been suggested ${ }^{26}$ but the exact relation between the two has not been clarified. In this case the patient had received antibiotics briefly prior to the onset of symptoms, so it is of course possible that this may be of relevance to the genesis of her colitis. We, however, do not feel that the colitis in this case was either an antibiotic associated colitis or an infectious (self limiting) colitis. Repeated stool cultures for pathogens and Clostridium difficile toxin were negative, and from the pathology point of view the picture in this case was unlike either. The neutrophil and crypt patterns were unlike self limiting colitis, and neither self limiting nor antibiotic associated colitis have a collagen band. In addition, both clinically and endoscopically these were not appropriate diagnoses.

Different coexisting diseases have been described in association with collagenous and lymphocytic colitis, including polyarteritis, ${ }^{26}$ seronegative arthritis, ${ }^{13} 27$ coeliac disease, ${ }^{6} 28-31$ small bowel villous atrophy unresponsive to gluten withdrawal, ${ }^{6} 1332$ pulmonary fibrosis, ${ }^{33}$ Raynaud's disease, ${ }^{34}$ and thyroid disease. ${ }^{101334}$ There is, however, no consistent association with these disorders, although a common autoimmune aetiology remains a possibility. ${ }^{35}$ We are unaware of any reported associations between scleroderma or primary biliary cirrhosis and collagenous or lymphocytic colitis.

Because there are no reported cases of either collagenous or lymphocytic colitis pursuing a fulminant course necessitating emergency colectomy, we wish to draw attention to a case of a woman presenting with collagenous colitis, confirmed with colonoscopic biopsies on two occasions, which did not settle with standard medical treatment and, because of a deteriorating clinical condition, subsequently necessitated a colectomy. The perioperative serosal appearance of the colon was of vascular congestion 
only, perhaps surprising in the light of the clinical features. An additional anomaly was the persistence of a normal erythrocyte sedimentation rate and serum albumin in a patient with torrential diarrhoea. Perhaps these features show that continuing conservative treatment in similar circumstances, despite failing medical treatment, may be appropriate.

This case also illustrates the dramatic lability of the collagen abnormality. After extensive sampling of the entire colon only a mild increase in intraepithelial lymphocytes was shown with no thickening of the collagen table whatsoever. It clearly shows that both the collagen and the colitis of collagenous colitis can wax and wane over short periods of time, and the pathogenesis of the diarrhoea may be independent of both, as suggested by the colonic secretion shown during in vivo perfusion studies. ${ }^{16}$ Collagen is usually regarded as stable and its lability during the natural history of collagenous colitis, in particular in this case where it was rapid and complete, is puzzling. Collagen types I and III have been shown in the abnormal band ${ }^{21} 36$ with type IV being restricted to the basal lamina. There is one report of type IV deposited within the band. ${ }^{15}$ As far as we are aware, however, there have been no studies on collagen turnover in collagenous colitis that allow speculation on an increased metabolism as a mechanism for these fluctuations. Finally, for the first time the interchange between the pathology of collagenous colitis and lymphocytic colitis is seen in a colectomy specimen free of the sampling error inherent in the existing studies in the literature. It highlights the overlapping picture and emphasises the difficulties faced in resolving the issue whether two aetiologically distinct diseases do exist. It is interesting that the intraepithelial lymphocyte count in this patient was still raised at the time of ileostomy revision, perhaps showing a permanent immunological defect.

We have, therefore, described the first case of collagenous colitis necessitating, on clinical grounds, a colectomy and present evidence supporting the claim that collagenous colitis and lymphocytic colitis are different manifestations of the same disease. We also have described, for the first time, an association with the CREST syndrome and primary biliary cirrhosis.

1 Lindstrom CG. 'Collagenous colitis' with watery diarrhoea - a new entity? Pathol Eur 1976; 11: 87-9.

2 Read NW, Krejs GJ, Read MG, Santa Ana CA, Morawski SG, Fordtran JS. Chronic diarrhoea of unknown origin. Gastroenterology 1980; 78: 264-71.

3 Sloth H, Bisgaard C, Grove A. Collagenous colitis: a prospective trial of prednisolone in six patients. F Intern Med 1991; 229: 443-6.

4 Lazenby AJ, Yardley JH, Giardiello FM, Jessurun J, Bayless TM. Lymphocytic ('microscopic') colitis: a comparative histopathologic study with particular reference to collagenous colitis. Hum Pathol 1989; 20: 18-28.

5 Farah DA, Mills PR, Lee FD, McLay A, Russell RI. Collagenous colitis: possible response to sulfasalazine and local steroid therapy. Gastroenterology 1985; 88: 792-7.

6 Kingham JGC, Levison BA, Ball JA, Dawson AM. Microscopic colitis - a cause of chronic watery diarrhoea. Microscopic colitis - a caus
$B M \mathcal{H} 1982 ; 285: 1601-4$.

7 Teglbjaerg PS, Thayssen EH, Jensen HH. Development of collagenous colitis in sequential biopsy specimens. Collagenous colitis in sequential
Gastroenterology $1984 ; 87: 703-9$.
8 Jessurun J, Yardley JH, Lee EL, Vendrell DD, Schiller LR, Fordtran JS. Microscopic and collagenous colitis: different names for the same condition? Gastroenterology different names fo

9 Yardley JH, Lazenby AJ, Giardiello FM, Bayless TM. Collagenous, 'microscopic', lymphocytic, and other more subtle forms of colitis. Hum Pathol 1990; 21: 1089-91.

10 Widgren S, Jlidi R, Cox N. Collagenous colitis: histologic, morphometric, immunohistochemical and ultrastructura studies: Report of 21 cases. Virchows Arch $(A) 1988 ; 413$ : 287-96.

11 Bogolometz WV, Flejou JF. Newly recognised forms of colitis: collagenous colitis, microscopic (lymphocytic) colitis, and lymphoid follicular proctitis. Semin Diagn Colitis, and lymphoid

12 Lindstrom CG. Collagenous colitis: an updating. Pathol Res Pract 1989; 185: A90.

13 Giardiello FM, Lazenby AJ, Bayless TM, Levine EJ, Bias WB, Ladenson PW, et al. Lymphocytic (microscopic) colitis. Clinicopathologic study of 18 patients and comparison to collagenous colitis. Dig Dis Sci 1989; 34: 1730-8.

14 Bo-Linn GW, Bendrell DD, Lee E, Fordtran JS. An evaluation of the significance of microscopic colitis in patients with chronic diarrhoea. 7 Clin Invest 1985; 75 1559-69.

15 Loo FD, Wood CM, Soergel KH, Komorowski RA, Cheung H, Gay S, et al. Abnormal collagen deposition and ion transport in collagenous colitis. Gastroenterology and ion transport

16 Rask-Madsen J, Hansen MGJ, Bukhave K, Scient C, Henrik-Nielson $R$. Colonic transport of water and electrolytes in a patient with secretory diarrhoea due to collagenous colitis. Dig Dis Sci 1983; 28: 1141-6.

17 Giardiello FM, Lazenby AJ, Yardley JH, Bias WB, Johnson $\mathrm{J}$, Alianiello RG, et al. Increased HLA A1 and diminished HLA A 3 in lymphocytic colitis compared to controls and patients with collagenous colitis. Dig Dis Sci 1992; 37: patients

18 Lee E, Schiller LR, Vendrell D, Santa Ana CA, Fordtran JS Subepithelial collagen table thickness in colon specimens from patients with microscopic colitis and collagenous from patients with microscopic colitis and
colitis. Gastroenterology 1992; 103: 1790-6.

19 Leigh C, Elahmady A, Mitros FA, Metcalf A, Al-Jurf A. Collagenous colitis associated with chronic constipation. Am $\mathcal{F}$ Surg Pathol 1993; 17: 81-4.

20 Carpenter HA, Tremaine WJ, Batts KP, Czaja AJ. Sequential histologic evaluations in collagenous colitis: correlations with disease behaviour and sampling strategy. Dig Dis Sci 1992; 37: 1903-9.

21 Jessurun J, Yardley JH, Giardiello FM, Hamilton SR, Bayless TM. Chronic colitis with thickening of the subepithelial collagen layer (collagenous colitis): histologic findings in 15 patients. Hum Pathol 1987; 18: 839-48.

22 Gubbins GP, Dekovich AA, Ma CK, B39-48. Collagenous colitis: report of nine cases and review of the Collagenous colitis: report of nine cases

23 Bogolometz WV. Collagenous colitis. Curr Top Pathol 1990; 81: 219-27.

24 Palmer KR, Berry H, Wheeler PJ, Williams CB, Fairclough $\mathrm{P}$, Morson BC, et al. Collagenous colitis - a relapsing and remitting disease. Gut 1986; 27: 578-80.

25 Jarnerot G, Tysk C, Bohr J, Eriksson S. Collagenous colitis and fecal stream diversion. Gastroenterology 1995; 109: 449-55.

26 Giardiello FM, Hansen C, Lazenby AJ, Hellman DB, Milligan FD, Bayless TM, et al. Collagenous colitis in setting of non-steroidal anti-inflammatory drugs and antisetting of non-steroidal anti-inflammat

27 Giardiello FM, Bayless TM, Jessurun J, Hamilton SR Bayless TM. Collagenous colitis: physiologic and histopathologic studies in seven patients. Ann Intern Med 1987; 106: 46-9.

28 O'Mahony S, Nawroz IM, Ferguson A. Coeliac disease and collagenous colitis. Postgrad Med $\mathcal{f}$ 1990; 66: 238-41.

29 Hamilton I, Sanders S, Hopwood D, Bouchier IAD. Collagenous colitis associated with small intestinal villous atrophy. Gut 1986; 27: 1394-8.

30 Breen EG, Farren C, Connolly CE, McCarthy CF. Collagenous colitis and coeliac disease. Gut 1987; 28: 364-6.

31 Eckstein RP, Dowsett JF, Riley JW. Collagenous enterocolitis: a case of collagenous colitis with involvement of colitis: a case of collagenous colitis with involvement of

32 Hwang WS, Kelly JK, Shaffer EA, Sylwestrowicz T, Klassen J. A novel enteropathy with partial villous atrophy, microscopic colitis and pemphigoid change. f Clin Gastroenterol 1989; 11: 216-9.

33 Wiener MD. Collagenous colitis and pulmonary fibrosis. f Clin Gastroenterol 1986; 8: 677-80

34 Wang KK, Perrault J, Carpenter HA, Schroeder KW, Tremaine WJ. Collagenous colitis: a clinicopathologic correlation. Mayo Clin Proc 1987; 62: 665-71.

35 Roubenoff R, Ratain J, Giardiello F, Hochberg MC, Bias $\mathrm{W}$, Lazenby AJ, et al. Collagenous colitis, enteropathic arthritis, and autoimmune diseases: results of a patient survey. F Rheumatol 1989; 16: 1229-32.

36 Flejou JP, Grimaud JA, Molas G, Baviera E, Potet F. Collagenous colitis: ultrastructural study and collagen immunotyping of four cases. Arch Pathol Lab Med 1984; 108: 977-82. 